\title{
Chromatin acetylation, $\beta$-amyloid precursor protein and its binding partner FE65 in DNA double strand break repair
}

\author{
Irena Szumiel ${ }^{1 \otimes}$ and Nicolas Foray ${ }^{2}$ \\ ${ }^{1}$ Centre for Radiobiology and Biological Dosimetry, Institute of Nuclear Chemistry and Technology, Warszawa, Poland; ${ }^{2}$ INSERM, U836, Groupe \\ de Radiobiologie, Institut des Neurosciences, Grenoble, France
}

\begin{abstract}
Among post-translational modifications of chromatin proteins taking place in DNA double strand break (DSB) repair, acetylation plays a prominent role. This review lists several facts and hypotheses concerning this process. Lack of acetyltransferase TIP60 (HIV-Tat interacting protein of $60 \mathrm{kDa}$ ) activity results in cells with defective DSB repair. The enzyme is present in the nucleus in a multimeric protein complex. TIP60 dependent activation of ATM (ataxia telangiectasia mutated kinase) is an early event in the response to DNA breakage. Other important acetylations are those of histones $\mathrm{H} 4$ and $\mathrm{pH} 2 \mathrm{AX}$. Correct reconstruction of the damaged site is critical for survival and prevention of genetic and epigenetic changes in the cell that may affect the function of its daughter cells. Recently, two proteins with previously unsuspected functions in DSB repair have been identified as active in this process: Alzheimer $\beta$-amyloid precursor protein (APP) and its binding partner FE65, $\beta$-amyloid precursor binding protein. Their participation in DSB repair in both neuronal and non-neuronal cells is related to acetylation carried out by the acetyltransferase complex. The same function is ascribed to heterochromatin protein 1 (HP1). So far, the relations (if any) between TIP60 activation by HP1 and by the FE65 complex remain unidentified.
\end{abstract}

Keywords: FE65, $\beta$-amyloid precursor protein, chromatin acetylation, Tip60 histone acetyltransferase, MOF acetyltransferase, heterochromatin protein 1, DNA double strand break repair

Received: 18 October, 2010; revised: 07 December, 2010; accepted: 21 January, 2011; available on-line: 15 March, 2011

\section{INTRODUCTION}

DNA double strand breaks (DSB) inflicted by ionising radiation, oxidants or topoisomerase II poisons belong to the most noxious types of DNA lesions, because they may lead to loss of genetic information. This may give rise to cancerogenesis and other pathogenic or lethal effects. Various aspects of DSB repair have been presented and discussed in numerous comprehensive review papers (Lisby \& Rothstein, 2009; Mahaney et al., 2009; Mimitou \& Symington, 2009; Nakamura et al., 2009; Pardo et al., 2009; Costes et al., 2010; Huertas 2010; Lieber, 2010). Its image becomes more complicated with the discovery of new participating proteins, which apparently are critical for efficient and error-free DSB repair (e.g., Lin et al., 2010). Our understanding of the details of end-joining of broken DNA chains by enzymes and helper proteins seems now to be almost complete, yet new functions and interactions are still being discovered.
A factor that should not be overlooked is that, in contrast with prokaryotes, DSB repair in eukaryotes takes place in the context of chromatin. Hence, its outcome is profoundly affected by the nuclear architecture. The processes of transcription-related chromatin modification, nucleo-cytoplasmic shuttling of activators, inhibitors or chaperones, and positioning within the nucleus also apply to DSB repair. These factors are especially important when the human response to ionising radiation and DNA damaging drugs is analysed, to optimize radiotherapy or prevent occupational hazard. It is significant that radiation sensitivity is a feature of laminopathies such as Hutchinson-Gilford progeria, and atypical Werner syndrome with mutations in nuclear lamin $\mathrm{A} / \mathrm{C}$ (Chen et al., 2003; Constantinescu et al., 2010). Progeroid mice show a considerable delay in DSB repair measured on the basis of $\gamma \mathrm{H} 2 \mathrm{AX}$ foci disappearance compared to that of wild type fibroblasts. When treated with a combination of statins and aminobisphosphonates that inhibited both farnesylation and geranylgeranylation of progerin and prelamin $\mathrm{A}$, the animals displayed a weakened aging-like phenotype as well as normal DSB repair rate (Varela et al., 2008).

Many recent papers concern structural alterations of chromatin in the vicinity of DSB creating a pattern of sequential modifications of chromatin proteins and resulting conformational changes that precede, accompany or follow every step of repair (reviewed by Pandita \& Richardson, 2009). The ultimate goal of that process is to restore the structure of the damaged site to its original state. These spatiotemporal chromatin alterations are critical not only for the restoration of DNA integrity but also for the coordination of repair with cell cycle control, and for the ultimate choice of the cell's fate. Here, the role of $\gamma \mathrm{H} 2 \mathrm{AX}$ foci is essential and their components sequentially associate and dissociate carrying out the functions necessary at each step of DSB repair (e.g., Nakamura et al., 2010).

\section{e-mail: i.szumiel@ichtj.waw.pl}

Abbreviations: $A B L$, Abelson tyrosine kinase; AICD, APP intracellular domain; APP, $\beta$-amyloid precursor protein; ATM, ataxia telangiectasia mutated kinase; DNA-PKcs, catalytic subunit of DNAdependent protein kinase; $C D K$, cyclin dependent protein kinase; DSB, DNA double strand break; FE65, $\beta$-amyloid precursor binding protein; H3K9me3, trimethylated lysine 9 of histone H3; H4K16, histone $\mathrm{H} 4$ lysine 16; HDAC, histone deacetylase; HP1, heterochromatin protein 1; HRR, homologous recombination repair; NHEJ, non-homologous end-joining; MOF, Drosophila male absent on the first; MRN, Mre11-RAD50-NBS1; PTB, phosphotyrosine binding domain; SH2, Src homology domain 2; TIP60, HIV Tat-interacting protein of $60 \mathrm{kDa}$; TRRAP, TRansformation/tRanscription domainAssociated Protein; WT, wild type; WW, tryptophane-rich, prolineproline-leucine-proline sequence binding domain 
Recently, two proteins with previously unsuspected functions in DSB repair have been identified as active in this process: Alzheimer $\beta$-amyloid precursor protein (APP) and its binding partner FE65, $\beta$-amyloid precursor binding protein. As discussed further in the text, their participation in repair is related to acetylation carried out by the acetyltransferase complex.

\section{THE IMPORTANCE OF ACETYLATION IN DSB REPAIR}

Each new review on DSB repair reports some freshly made observations and views on the sequence of events starting with damage infliction and ending with the restoration of the original state of the particular chromosome fragment that was damaged. It is now clear that some of the processes involved differ depending on whether the site of the damage is in euchromatin or heterochromatin (Goodarzi et al., 2009; Noon et al., 2010), on the cell cycle phase, the repair path choice and crosstalk, and on the cell genotype and phenotype. Canonical or alternative NHEJ (non-homologous end-joining) and HRR (homologous recombination repair) use different sets of proteins, nevertheless, some general principles of chromatin remodelling preceding and following the repair step are similar.

Among the post-translational modifications of chromatin proteins taking place in DSB repair, acetylation plays a prominent role. Therefore, in the simplified diagram showing chromatin remodelling steps (Fig. 1) emphasis was put on this post-translational modification. Other modifications are equally important for the outcome of DNA repair and their full list is given in a recent review (Pandita \& Richardson 2009).

One of the main enzymes acting in the damaged cell is a transcription co-activator, acetyltransferase TIP60. Lack of its activity results in defective DSB repair and apoptosis (Ikura et al., 2000). Also, its function is required for the assembly of $\gamma \mathrm{H} 2 \mathrm{AX}$ repair foci (Chailleux et al., 2010). The enzyme participates in various repair steps and is present in the nucleus in a multimeric

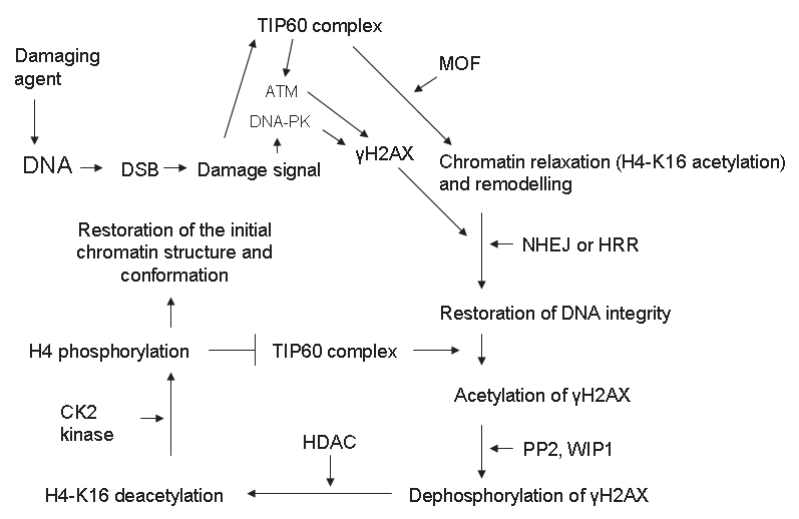

Figure 1. The sequence of events that take place in chromatin upon DSB infliction and the subsequent repair shown in a simplified way

Post-translational modifications are omitted with the exception of acetylation and dephosphorylation where it precedes deacetylation. The diagram is based on the review of Altaf et al. (2007) with modifications, Moon et al. (2010), Sharma et al. (2010). H4-K16, histone $\mathrm{H} 4$ acetylated at lysine 16; WIP1, wild-type p53-induced phosphatase 1 and PP2 protein phosphatase 2, both directly dephosphorylate $\mathrm{YH} 2 \mathrm{AX}$; TIP60 and MOF-acetyltransferases (see text for detailed discussion). protein complex known as the evolutionarily conserved NuA4 complex (Doyon \& Cote, 2004). The review of Sapuntzi et al. (2006) lists 18 components of the human TIP60 complex. Also in cells missing TRRAP (TRansformation/tRanscription domain-Associated Protein, component of the complex) DSB repair by homologous recombination is impaired and this is accompanied by diminished acetylation of histone $\mathrm{H} 4$ which normally takes place at the damaged site (reviewed by Lee \& Workman, 2007; Murr et al., 2007). The repair defect resulting from TRRAP absence can be corrected by treatment with agents that relax chromatin, e.g., by hypotonic medium (Murr et al. 2006; 2007). This points to the importance of a decompacted chromatin conformation for DSB repair, associated with the access of the repair machinery to chromatin at the damaged site. As mentioned further in the text, more than access is at stake.

The TIP60-associated complex shows ATPase, DNA helicase, and DNA binding activities and interacts with some of the repair enzymes (Ikura et al., 2000; Squatrito et al., 2006; Sun et al., 2010). As found recently (Robert et al., 2006; Chailleux et al., 2010), TIP60-associated TRRAP mediates formation of a complex with the DSB sensor MRN (MRE11-RAD50-NBS1). It differs from the transcription active complex in that it does not contain p400, whereas it contains some still unidentified proteins (Chailleux et al., 2010). TIP60 dependent activation of ATM (ataxia telangiectasia mutated kinase) and DNA-PKcs (catalytic subunit of DNA-dependent protein kinase) is an early event in the response to DNA breakage (Jiang et al., 2006; review in Squatrito et al., 2006). Most probably it is carried out by TIP60-MRN, whereas further acetylations, those of lysine 16 residues on histone $\mathrm{H} 4$ related to chromatin decompaction at the DSB, take place with the participation of the transcription active TIP60 complex. Also histone $\gamma \mathrm{H} 2 \mathrm{AX}$ is acetylated by that complex. The latter modification precedes its exchange and dephosphorylation, thus "erasing the $\gamma \mathrm{H} 2 \mathrm{AX}$ mark" (Squatrito et al., 2006) after DSB repair is completed. These findings indicate that acetylation is important for the correct reconstruction of the damaged site. This, in turn, is critical for survival and for the lack of genetic and epigenetic changes in the cell that may affect the function of its daughter cells.

Recently, another histone acetyltransferase has attracted attention in connection with DNA damage response: the MOF (MYST1) acetyltrasferase (Gupta et al., 2005; Sharma et al., 2010; Taipale et al., 2005). Mammalian MOF is the ortholog of the Drosophila male absent on the first (MOF) protein, a component of the $\mathrm{X}$ chromosome dosage compensation system. It is responsible for histone $\mathrm{H} 4$ lysine 16 (H4K16) acetylation in the chromatin decondensation step associated with transcription activation. It also acetylates lysine 120 of the transcription factor $\mathrm{p} 53$, a modification important for the activation of the transcription of pro-apoptotic genes (Taipale et al., 2005; Li et al., 2009; Cai et al., 2010). Thomas et al. (2008) found that MOF is necessary for mouse embryo development and maintenance of the chromatin architecture and in these respects MOF is functionally nonredundant with TIP60. Importantly, according to Gupta et al. (2005), MOF interacts with ATM and this is necessary for its activation possibly because hMOF may act as a transducer of chromatin structural alterations to ATM after DNA damage.

MOF depletion considerably decreases DSB repair both by NHEJ and HRR (Sharma et al. 2010). This points to a defect in the chromatin decondensation step, 
necessary for the functioning of both repair systems. The HRR defect is revealed as a reduced frequency of RAD51 foci and inefficient recruitment of RAD52 on the damaged chromatin. Additionally, a NHEJ specific effect also follows MOF depletion, as MOF is associated with DNA-PKcs. ATM-dependent ionising radiation-induced phosphorylation of DNA-PKcs is also abrogated in MOF-depleted cells. At the cellular level, a deficiency in MOF results in increased residual DSB level and chromosomal aberration frequency (Gupta et al., 2005).

Both MOF and TIP60 are members of the MYST superfamily of acetyltransferases, which took its name from its four "founding members": human MOZ (monocytic leukemia zinc finger protein), yeast Ybf2 (renamed Sas3, for something about silencing 3), yeast Sas2 and mammalian TIP60. The relation between MOF and TIP60 functions in the cellular response to DNA damage is unclear. It is possible that they may replace each other depending on the expression level at different stages of embryonal development and in adult tissues (Thomas et al., 2007).

\section{HISTONE DEACETYLASES AND DSB REPAIR}

The level of acetylation of histones is controlled by the balance between the action of histone acetyltransferases and deacetylases (HDACs). Eleven mammalian HDACs have been identified and divided into four classes (De Ruijter et al., 2003; Eot-Houllier et al., 2009). They are functionally diverse and heterogenous as concerns both substrate specificity and localization: class I HDAC are only nuclear; class II HDAC can traffic between the cytoplasm and nucleus in response to specific signals. For example, HDAC4 and 5 (class II) are retained in the cytoplasm depending on the activity of the 14-3-3 anchoring protein; the nuclear export of HDAC5 depends on its phosphorylation by calcium/calmodulin-dependent protein kinase (reviewed by Khochbin et al., 2001). Class III HDAC (also called sirtuins) are $\mathrm{NAD}^{+}$-dependent protein deacetylases and their function is related to the redox status of the cell. HDAC11 belongs to class IV which shares structure features of both class I and II enzymes. Interestingly, SIRT1 seems to be the most specific class III HDAC for deacetylation of H4K16, the key acetylation seen in the response to DNA damage (Hajji et al., 2010).

Apart from the removal of acetyl groups from the chromatin core histones, both the nuclear and cytoplasmic HDACs deacetylate other proteins. Hence, the use of HDAC inhibitors for studying the effects of combination radio- and chemotherapy creates results that are very difficult to interpret from the point of view of molecular mechanisms. In the case of DNA damage and repair, HDAC inhibition can increase the initial damage due to modified DNA shielding by chromatin proteins, alter the expression of DNA repair proteins or their activity by acetylation increase and, last but not least, disturb the pattern of DNA damage histone code (reviewed by Eot-Houllier et al., 2009). Also, the altered localization of HDAC may be a result of inhibitor treatment as is the case of LBH589, a cinnamic hydroxamic acid analogue. Pre-treatment followed by X-irradiation increases the duration of $\gamma \mathrm{H} 2 \mathrm{AX}$ foci and apoptosis and also confines HDAC4 to the cytoplasm in non-small cell lung cancer cells, whereas X-irradiation alone causes nuclear translocation of HDAC4 (Geng et al., 2006).

\section{STRUCTURE AND FUNCTION OF APP AND FE65}

Less known partners of the TIP60 complex are APP and its binding partner FE65. APP and FE65 have been known for about 10 years as transcription activators (Cao \& Suedhof, 2001; review in Słomnicki \& Leśniak, 2008). Their biological properties have been studied in the context of Alzheimer disease and their participation in DSB repair has only recently been revealed (Minopoli et al., 2007).

APP and two related proteins, APPLP1 and APPLP2, are membrane glycoproteins (Reinhardt et al., 2005). Their cleavage by secretases generates various peptide fragments, among them the $\beta$-amyloid peptides which form the senile plaques characteristic of Alzheimer disease. Another cleavage product is a short cytosolic fragment, known as APP intracellular domain (AICD). Figure 2 shows the structure of APP, interaction motifs in the intracellular domain and a list of some interacting proteins, among them FE65 (Fiore et al., 1995; Borg et al., 1996). Five out of eight potential phosphorylation sites (Lee et al., 2003) located within AICD are placed in the interaction motifs. In such cases, phosphorylation may affect the binding of partner proteins (reviewed by Tamayev et al., 2009). For example, phosphorylated Tyr-682 enables the binding of cytosolic tyrosine kinases (among them Abelson tyrosine kinase, ABL) and proteins with SH2 domain (Src homology domain 2) like SHC A, B and C. Phosphorylated Thr-668 blocks interaction with 14-3-3 $\gamma$ while being indispensable for FE65 binding. The phosphorylation status of the AICD domain profoundly affects the cellular functions of APP

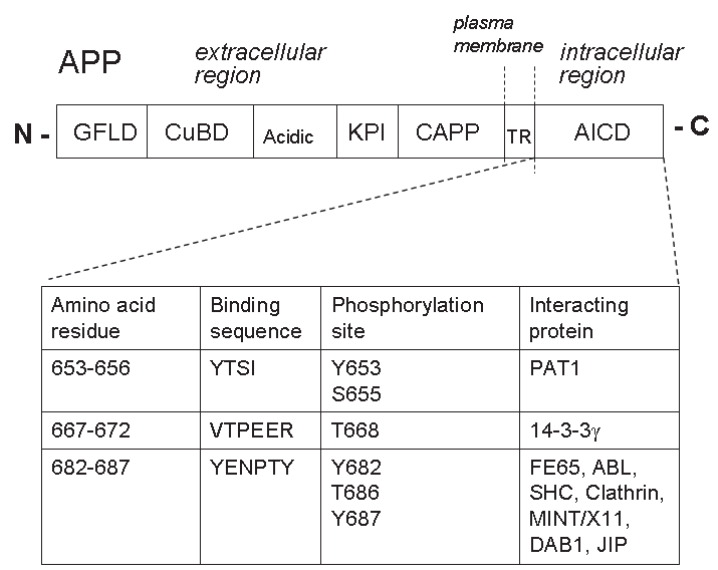

Figure 2. APP domain structure (drawn not to scale) The extracellular part of APP consists of the N-terminal growth factor-like domain (GFLD) and the copper-binding domain (CuBD). These domains are linked via the acidic region to the Kunitz-type protease inhibitor domain (KPI) and a cysteine-poor, conserved sequence (central APP domain, CAPP-domain) which contains two N-glycosylation sites. The linker connects the extracellular domains to the transmembrane region (TR) and the APP intracellular C-terminal domain (AICD). The binding motifs of AICD are shown and some interacting proteins listed (also discussed in the text). 14-3-3y, member of the 14-3-3 adaptor protein family; $A B L$, cytosolic tyrosine kinase; DAB1, disabled homologue 1, a cytoplasmic adaptor protein that regulates actin cytoskeleton in neuronal migrations during mammalian brain development; JIP, JNK-interacting protein (JNK- C-Jun N-terminal kinase); MINT/X11, a family of Munc18-interacting adaptor proteins in synaptic vesicle exocytosis; Pat1, protein interacting with APP tail-1; SHC, Src homologous-collagen homologue, adaptor protein involved in the coupling of activated tyrosine kinase growth factor receptors to the Ras activation pathway. 


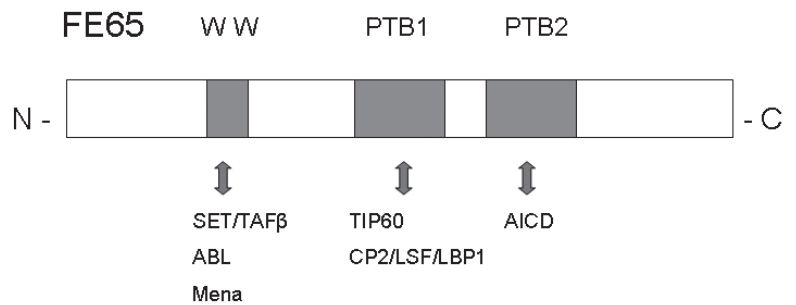

Figure 3. AICD domain structure (not drawn to scale)

The list of interacting proteins is based on the review of Słomnicki \& Leśniak (2008). WW, tryptophane-rich, proline-proline-leucineproline sequence binding domain; PTB phosphotyrosine binding domain; CP2/LSF/LBP1, transcription factor; Mena, mammalian homologue of the Drosophila actin cytoskeleton regulatory protein enabled; TIP60, acetyltransferase (Tat interactive protein-60).

(detailed reviews in Müller et al., 2008; Tamayev et al., 2009).

FE65 essentially is known as a neural adaptor protein interacting with a number of proteins, including APP; however, it is present in other cell types. As shown in Fig. 3, it contains one WW (tryptophane-rich, prolineproline-leucine-proline sequence binding) domain (Ermekova et al., 1997) and two PTB (phosphotyrosine binding) domains (review in Russo et al., 1998). The WW domain binds the ABL kinase (Perkinton et al., 2004). Also the nucleosome assembly factor SET/TAF1 $\beta$ and the mammalian homologue of the Drosophila actin cytoskeleton regulatory protein enabled (Mena) interact with FE65 WW domain (Ermekova et al., 1997; Telese et al., 2005). The PTB1 domain binds among others TIP60 (Cao \& Suedhof, 2001) and the transcription factor CP2/LSF/ LBP1 (Zambrano et al., 1998), whereas PTB2 interacts with the APP intracellular domain (Fiore et al., 1995).

Numerous cellular functions of APP and its proteolysis product, AICD, alone or in complex with FE65 have been identified (e.g., Williamson et al., 2002; Sondag et al., 2004; Lopez-Lopez et al., 2007; Minopoli et al., 2007; Giliberto et al., 2008; Nikolaev et al., 2009; Vázquez et al., 2009). In particular, modulation of gene expression, apoptosis and regulation of actin cytoskeleton function have been reviewed by Müller et al. (2008). The recently identified function of AICD and FE65 in DSB repair is presented further in the text. The functional diversity of both proteins can be explained by their modular structure enabling interactions with various proteins, thus producing scaffolds for the proper spatial arrangement of active enzyme complexes.

\section{AICD AND FE65 AS TRANSCRIPTION MODULATORS}

Activation of transcription was initially ascribed to AICD and FE65 bound to the transcription factor CP2/LSF/LBP1 (Zambrano et al., 1998) or complexed with TIP60 (Cao \& Suedhof, 2001; von Rotz et al., 2004). However, further studies brought discrepancies and disagreement concerning the detailed mechanism of nuclear translocation and activation of the participating proteins (review by Müller et al., 2008). The most often accepted model is that of AICD release by $\gamma$-secretase, induction of an open conformation of FE65, followed by nuclear translocation of both proteins separately (Nakaya \& Suzuki, 2006) or bound together (Stante et al., 2009). FE65 seems to be essential for the nuclear translocation of AICD, and AICD mutated at the FE65 interaction site remains largely cytosolic (Kimber- ly et al., 2001; Kinoshita et al., 2002a, 2002b). In contrast to FE65, the $\mathrm{X} 11 \alpha$ adaptor protein arrests AICD within the cytosol (von Rotz et al., 2004). Binding of FE65 or both proteins to TIP60 activates its histone acetyltransferase function necessary to produce a locally relaxed chromatin conformation, a requirement for further steps of transcription. Which protein is the "true" activator remains uncertain, and there are several models of AICD and FE65 function in transcription activation, as discussed below.

Whether FE65 is a transport protein or a necessary component of the TIP60 complex is a matter of controversy. In the model of Cao and Suedhof (2004), AICD remains at the plasma membrane as a part of APP and serves to induce the open conformation of FE65. Upon translocation to the nucleus, FE65 binds to and activates TIP60. Another model, also supported by convincing experimental data, is that of Hass and Yankner (2005). According to those authors, APP and Fe65 activate transcription even in cells that are unable to generate AICD or are treated with $\gamma$-secretase inhibitor. The explanation is that APP recruits TIP60 to the plasma membrane, where TIP60 becomes phosphorylated by a cyclin dependent kinase (CDK) and binds to FE65. Thus activated and stabilized, TIP60 returns to the nucleus, remaining bound to FE65. A CDK inhibitor, roscovitine, blocks TIP60 activation, whereas treatment with leptomycin B considerably decreases activation by inhibiting TIP60 export to the cytoplasm, thus preventing its contact with APP. This effect and microscopic co-localization images of APP and TIP60 at the plasma membrane support the concept of TIP60 shuttling between the membrane and nuclear compartments.

It should be added that these different models are supported by well documented experimental data and the reasons for the discrepancies remain, so far, unclear. Nevertheless, there is enough evidence that the function of FE65 and AICD in the nucleus is linked to TIP60 both in transcription and DSB repair, as described in the next section.

\section{AICD AND FE65 IN DSB REPAIR}

The first observation indicating a role of FE65 in DNA repair was made by Minopoli et al. (2007): mouse embryo fibroblasts derived from FE65 knockout mice were more sensitive to DNA damaging agents than the wild type cells. This effect was reversed by FE65 expression but not by an FE65 transfectant with a MAPK-derived nuclear export sequence (NES) unable to accumulate in the nucleus (Minopoli et al., 2007). When either full FE65 or a $\triangle$ PTB1 mutant unable to interact with TIP60 were used for transfection, only the latter did not abrogate the high sensitivity of FE65 knockout mouse embryo fibroblasts to etoposide (the sensitivity measure was tail moment in the comet assay).

Furthermore, in FE65 null cells TIP60/TRRAP recruitment to the damaged DNA sites as well as TIP60dependent histone $\mathrm{H} 4$ acetylation were significantly decreased. Simultaneously, DNA repair efficiency was markedly lower. These observations were made in NIH GS cells stably transfected with a tamoxifen-inducible form of the I-SceI restriction enzyme that produced DSB. Chromatin was then immunoprecipitated (ChIP) with TIP60 or acetylated histone $\mathrm{H} 4$ antibody and analysed with the use of real time PCR (polymerase chain reaction) (Stante et al., 2009). 


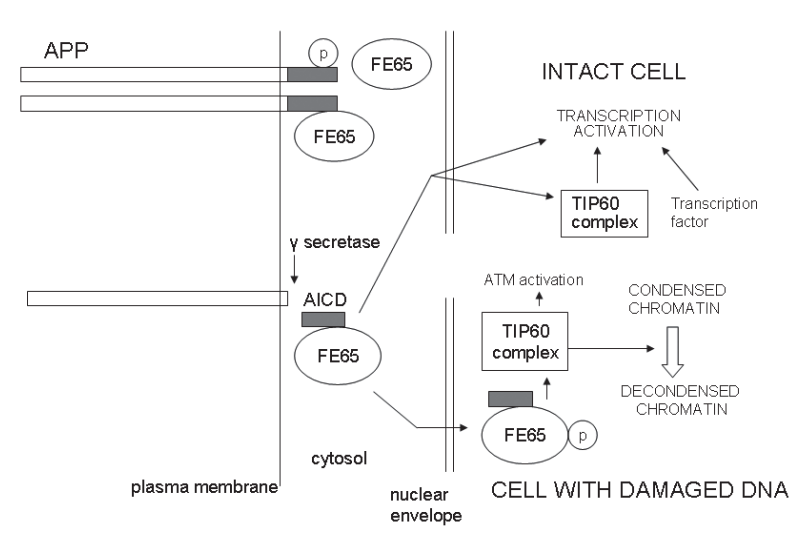

Figure 4. Model of APP and FE65 function in DSB repair The model (in the lower part of the diagram) is according to Minopoli et al. (2007) and Stante et al. (2009) and is compared to the function of APP and FE65 in the intact cell (see text for discussion).

An FE65-AICD interaction was required for the DNA repair-stimulating effect. This was indicated by demonstrating that AICD suppression caused a significant decrease in TIP60/TRRAP recruitment to the I-SceI endonuclease induced breaks, in the extent of histone H4 acetylation induced by DNA cleavage, as well as in the DNA repair efficiency. Further support for the role of AICD came from the observation that in contrast to WT FE65, an FE65 mutant (C655F) unable to interact with AICD did not bind to chromatin. WT FE65 was associated with chromatin both in undamaged cells and those treated with DNA-damaging agents (Stante et al., 2009).

Although there are still further details to be established, the observations of Minoli et al. (2007) and Stante et al. (2009) presented above are sufficient to support a hypothetical model of function of the AICD-FE65 complex in DSB repair shown diagrammatically in Fig. 4. In the intact cell, there is a basal level of AICD which is either degraded or binds FE65 thus favouring its nuclear translocation. In the damaged cell (upon treatment with $\mathrm{X}$-rays, hydrogen peroxide or topoisomerase II poisons) in a few minutes after DNA damage infliction, nuclear FE65 becomes phosphorylated (Minopoli et al., 2007). In parallel, $\gamma$-secretase cleaves APP, thus producing an additional amount of AICD. The mechanism of activation and the nature of the signal triggering this response remain to be established. The activity of $\gamma$-secretase is a possible regulatory factor involved in maintaining the balance between cytosolic and nuclear FE65 and AICD (Kinoshita et al., 2002a; 2002b). Since $\gamma$-secretase activation can be mediated by JNK (Liao et al., 2004) it is plausible that $\mathrm{X}$-irradiation may indirectly activate this protease, because JNK is activated by this damaging agent (Narang et al., 2009).

Although there is still much to be discovered about the function of FE65 in DSB repair, it seems that it is an important part of the molecular machine that acts in the damaged cell at different steps of chromatin remodelling and recruiting the repair proteins in a concerted way. FE65 interacts with oncoprotein SET/TAF-I $\beta$ which is a subunit of the complex that inhibits histone acetylation (Seo et al., 2001; 2002). It has been noted that induction of histone acetylation promotes demethylation of ectopically methylated DNA (Cervoni \& Szyf, 2001), whereas overexpression of SET/TAF-I $\beta$ inhibits demethylation (Cervoni et al., 2002). SET/TAF-I $\beta$ also plays the role of histone chaperone with nucleosome assembly and disassembly activities (Muto et al., 2007) and inhibits protein phosphatase 2 (Li et al., 1996). The latter dephosphorylates histone $\gamma \mathrm{H} 2 \mathrm{AX}$, a step required during disassembly of $\gamma \mathrm{H} 2 \mathrm{AX}$ foci. So, FE65 may indirectly be involved in the regulatory role of SET/TAF-I $\beta$ in the post-repair steps of chromatin modifications and in correlating epigenetic states of histones and DNA, both important for the proper reconstruction of the damaged site following DSB repair.

\section{TIP60 ACTIVATION AND HETEROCHROMATIN PROTEIN 1}

One may wonder how general the mechanism of TIP60 activation described above is. Is it related to the choice of the DNA repair pathway? Is it cell type specific? Answers to these questions may explain some of the differences in experimental results that lead to a variety of models of AICD/FE65 action. Interestingly, an alternative mechanism of TIP60 activation has recently been proposed.

Sun et al. (2009; 2010) describe TIP60 activation based on a totally different principle. Their model takes into consideration the fact that both TIP60 and HP1 (heterochromatin protein 1) chromodomains recognise trimethylated lysine 9 of histone $\mathrm{H} 3$ ( $\mathrm{H} 3 \mathrm{~K} 9 \mathrm{me} 3$ ) residues. Ayoub et al. (2008) report that in response to DNA damage HP1 transiently dissociates from chromatin at the damaged sites. Upon damage infliction, CK2 kinase phosphorylates Thr-51 localized in the chromodomain of HP1 thus disrupting its interaction with $\mathrm{H} 3 \mathrm{~K} 9 \mathrm{me} 3$. The model of Sun et al. (2010) predicts that an inactive ATM-TIP60 complex is recruited to the DSB by MRN, and this enables interaction between TIP60 and H3K9me3 previously occupied by HP1. The interaction acts as an allosteric regulator of the acetyltransferase activity, leading to ATM acetylation and activation. The model is based on several experimental observations listed by Sun et al. (2009; 2010). Significantly, inactivating mutations in the HP1 chromodomain prevent both the interaction between TIP60 and H3K9me3 and the upregulation of acetyltransferase activity by DNA damage. This inhibits the subsequent acetylation and activation of ATM kinase. Furthermore, reduction of $\mathrm{H} 3 \mathrm{~K} 9$ methylation in chromatin increases radiation sensitivity and genomic instability (Sun et al., 2009). Three isoforms of HP1, considered the hallmark of heterochromatin, interact with histones, transcriptional co-repressors and DNA replication factors and are also present in euchromatin (Kwon \& Workman, 2008). Therefore, it is difficult to reconcile the release of $\mathrm{HP} 1$ from $\mathrm{H} 3 \mathrm{~K} 9 \mathrm{me} 3$ residues, unevenly distributed within chromatin, with activation of TIP60-ATM taking place at randomly inflicted DSB. On the other hand, as already mentioned, the DSB sensor complex MRN directs TIP60 to its site of action by interaction with TRRAP (Chailleux et al., 2010). The presence of $\mathrm{H} 3 \mathrm{~K} 9 \mathrm{me} 3$ at that site would be fortuitous.

\section{CONCLUDING REMARKS}

Observations concerning HP1 in cells with damaged DNA modify our views on the genomic "alarm system". DNA damage infliction (not only DSB but also UV and oxidative lesions) is followed by recruitment of HP1 to the damaged sites (Luijsterburg et al., 2009; Zarębski et al., 2009). Thus, MRN would be the DSB sensor, whereas HP1 would act as a universal sensor, switching on an 


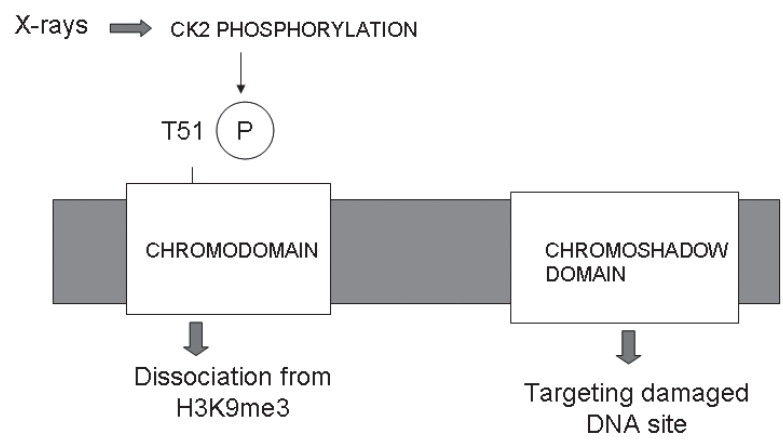

Figure 5. Domain structure of HP1 and functions induced by X-irradiation

Alterations following X-irradiation include phosphorylation of threonine 51 by CK2 kinase, loss of the capability of binding H3K9me3 (trimethylated lysine 9 of histone H3) and acquired capability to target the damaged DNA site (see text for discussion).

alarm signal for various types of DNA lesions. It now seems that upon DNA damage infliction, after the initial dissociation from $\mathrm{H} 3 \mathrm{~K} 9 \mathrm{me} 3$, HP1 re-associates with chromatin independently of $\mathrm{H} 3 \mathrm{~K} 9 \mathrm{me} 3$ and the HP1 chromodomain. The latter becomes phosphorylated on Thr-51 (T51-P) and this is expected to transiently relax heterochromatin or create an interaction surface with DNA repair proteins containing BRCT (C-terminal domain of the breast cancer susceptibility protein) or FHA (forkhead-associated) domains specifically recognising T51-P (Luijsterburg et al., 2009; reviewed by Ayoub et al., 2009; Dinant \& Luijsterburg, 2009).

So, HP1 recruitment occurs independently of its ability to associate with $\mathrm{H} 3 \mathrm{~K} 9 \mathrm{me} 3$ involving chromodomain and dependent on the phosphorylation status of Thr-51. In contrast, it is dependent on the chromoshadow domain (cf. Fig. 5). Ball and Yokomori (2009) discussing the contribution of HP1 to DNA repair mention the possibility that repair of DSB localized in heterochromatin may depend on HP1 in contrast with that in euchromatin. They recall that siRNA-mediated depletion of all three HP1 variants in mammalian cells exerts a negative effect on DSB repair only with a simultaneous ATM depletion and the latter is necessary for DSB repair in heterochromatin (Goodarzi et al., 2008). This contrasts with the clear effect of FE65/AICD on DSB repair reported by Minoli et al. (2007) and Stante et al. (2009).

The relations between the two routes of TIP60 activation are so far unknown. Both models need further experimental support. Whether TIP60 activation by FE65/ AICD is restricted in vivo to some cell types only or to certain nuclear compartments, remains to be established. Further research may open new perspectives on our understanding of the early steps of the cellular response to DNA damaging factors.

\section{Acknowledgements}

I.S. is supported by the statutory grant of the Ministry of Science and Higher Education to the Institute of Nuclear Chemistry and Technology. N.F. acknowledges support by Electricité de France (EDF) and Centre National d'Etudes Spatiales (CNES).

\section{REFERENCES}

Altaf M, Saksouk N, Côté J (2007) Histone modifications in response to DNA damage. Mutat Res 618: 81-90.
Ayoub N, Jeyasekharan AD, Bernal JA, Venkitaraman AR (2008) HP1beta mobilization promotes chromatin changes that initiate the DNA damage response. Nature 453: 682-686.

Ayoub N, Jeyasekharan AD, Venkitaraman AR (2009) Mobilization and recruitment of HP1, a bimodal response to DNA breakage. Cell Cycle 8: 2945-2950.

Ball AR Jr, Yokomori K (2009) Revisiting the role of heterochromatin protein 1 in DNA repair. I Cell Biol 185: 573-575.

Borg JP, Ooi J, Levy E, Margolis B (1996) The phosphotyrosine interaction domains of X11 and FE65 bind to distinct sites on the YENPTY motif of amyloid precursor protein. Mol Cell Biol 16: 6229-6241.

Cai Y, Jin J, Swanson SK, Cole MD, Choi SH, Florens L, Washburn MP, Conaway JW, Conaway RC (2010) Subunit composition and substrate specificity of a MOF-containing histone acetyltransferase distinct from the male-specific lethal (MSL) complex. I Biol Chem 285: 4268-4272.

Cao X, Südhof TC (2001) A transcriptionally [correction of transcriptively] active complex of APP with Fe65 and histone acetyltransferase Tip60. Science 293: 115-120.

Cao X, Südhof TC (2004) Dissection of amyloid-beta precursor protein-dependent transcriptional transactivation. I Biol Chem 279: 24601-24611.

Cervoni N, Szyf M (2001) Demethylase activity is directed by histone acetylation. I Biol Chem 276: 40778-40787.

Cervoni N, Detich N, Seo SB, Chakravarti D, Szyf M (2002) The oncoprotein Set/TAF-1beta, an inhibitor of histone acetyltransferase, inhibits active demethylation of DNA, integrating DNA methylation and transcriptional silencing. I Biol Chem 277: 25026-25031.

Chen L, Lee L, Kudlow BA, Dos Santos HG, Sletvold O, Shafeghati Y, Botha EG, Garg A, Hanson NB, Martin GM, Mian IS, Kennedy BK, Oshima J (2003) LMNA mutations in atypical Werner's syndrome. Lancet 362: 440-445.

Chailleux C, Tyteca S, Papin C, Boudsocq F, Puget N, Courilleau C, Grigoriev M, Canitrot Y, Trouche D (2010) Physical interaction between the histone acetyl transferase Tip60 and the DNA doublestrand breaks sensor MRN complex. Biochem J 426: 365-371.

Constantinescu D, Csoka AB, Navara CS, Schatten GP (2010) Defective DSB repair correlates with abnormal nuclear morphology and is improved with FTI treatment in Hutchinson-Gilford progeria syndrome fibroblasts. Exp Cell Res 316: 2747-2759.

Costes SV, Chiolo I, Pluth JM, Barcellos-Hoff MH, Jakob B (2010) Spatiotemporal characterization of ionizing radiation induced DNA damage foci and their relation to chromatin organization. Mutat Res 704: 78-87.

de Ruijter AJ, van Gennip AH, Caron HN, Kemp S, van Kuilenburg AB (2003) Histone deacetylases (HDACs), characterization of the classical HDAC family. Biochem J 370: 737-749.

Dinant C, Luijsterburg MS (2009) The emerging role of HP1 in the DNA damage response. Mol Cell Biol 29: 6335-6340.

Doyon Y, Côté J (2004) The highly conserved and multifunctional NuA4 HAT complex. Curr Opin Genet Dev 14: 147-154.

Eot-Houllier G, Fulcrand G, Magnaghi-Jaulin L, Jaulin C (2009) Histone deacetylase inhibitors and genomic instability. Cancer Lett 274: 169-176.

Ermekova KS, Zambrano N, Linn H, Minopoli G, Gertler F, Russo T, Sudol M (1997) The WW domain of neural protein FE65 interacts with proline-rich motifs in Mena, the mammalian homolog of Drosophila enabled. I Biol Chem 272: 32869-32877.

Fiore F, Zambrano N, Minopoli G, Donini V, Duilio A, Russo T (1995) The regions of the Fe65 protein homologous to the phosphotyrosine interaction/phosphotyrosine binding domain of She bind the intracellular domain of the Alzheimer's amyloid precursor protein. J Biol Chem 270: 30853-30856.

Geng L, Cuneo KC, Fu A, Tu T, Atadja PW, Hallahan DE (2006) Histone deacetylase (HDAC) inhibitor LBH589 increases duration of gamma-H2AX foci and confines HDAC4 to the cytoplasm in irradiated non-small cell lung cancer. Cancer Res 66: 11298-11304.

Giliberto L, Zhou D, Weldon R, Tamagno E, De Luca P, Tabaton M, D'Adamio L (2008) Evidence that the amyloid beta precursor protein-intracellular domain lowers the stress threshold of neurons and has a "regulated" transcriptional role. Mol Neurodegener 3: 12.

Goodarzi AA, Noon AT, Deckbar D, Ziv Y, Shiloh Y, Löbrich M, Jeggo PA (2008) ATM signaling facilitates repair of DNA doublestrand breaks associated with heterochromatin. Mol Cell 31: 167177.

Goodarzi, AA, Noon AT, Jeggo PA (2009) The impact of heterochromatin on DSB repair. Biochem Soc Trans 37: 569-576.

Gupta A, Sharma GG, Young CS, Agarwal M, Smith ER, Paull TT, Lucchesi JC, Khanna KK, Ludwig T, Pandita TK (2005) Involvement of human MOF in ATM function. Mol Cell Biol 25: 52925305 .

Hajji N, Wallenborg K, Vlachos P, Füllgrabe J, Hermanson O, Joseph B (2010) Opposing effects of hMOF and SIRT1 on H4K16 acetylation and the sensitivity to the topoisomerase II inhibitor etoposide. Oncogene 29: 2192-2204. 
Hass MR, Yankner BA (2005) A secretase-independent mechanism of signal transduction by the amyloid precursor protein. J Biol Chem 280: 36895-36904.

Huertas P (2010) DNA resection in eukaryotes, deciding how to fix the break. Nat Struct Mol Biol 17: 11-16.

Ikura T, Ogryzko VV, Grigoriev M, Groisman R, Wang J, Horikoshi M, Scully R, Qin J, Nakatani Y (2000) Involvement of the TIP60 histone acetylase complex in DNA repair and apoptosis. Cell 102: 463-473.

Kataoka Y, Murley JS, Baker KL, Grdina DJ (2007) Relationship between phosphorylated histone $\mathrm{H} 2 \mathrm{AX}$ formation and cell survival in human microvascular endothelial cells (HMEC) as a function of ionizing radiation exposure in the presence or absence of thiol-containing drugs. Radiat Res 168: 106-114.

Khochbin S, Verdel A, Lemercier C, Seigneurin-Berny D (2001) Functional significance of histone deacetylase diversity. Curr Opin Genet Dev 11: 162-166.

Kimberly WT, Zheng JB, Guénette SY, Selkoe DJ (2001) The intracellular domain of the beta-amyloid precursor protein is stabilized by Fe65 and translocates to the nucleus in a notch-like manner. $J$ Biol Chem 276: 40288-40292.

Kinoshita A, Whelan CM, Smith CJ, Berezovska O, Hyman BT (2002a) Direct visualization of the gamma secretase-generated carboxylterminal domain of the amyloid precursor protein, association with Fe65 and translocation to the nucleus. I Neurochem 82: 839-847.

Kinoshita A, Whelan CM, Berezovska O, Hyman BT (2002b) The gamma secretase-generated carboxyl-terminal domain of the amyloid precursor protein induces apoptosis via Tip60 in H4 Cells. J Biol Chem 277: 28530-28536.

Kwon SH, Workman JL (2008) The heterochromatin protein 1 (HP1) family, put away a bias toward HP1. Mol Cell 26: 217-227.

Lee KK, Workman JL (2007) Histone acetyltransferase complexes, one size doesn't fit all. Nat Rev Mol Cell Biol 8: 284-295.

Lee MS, Kao SC, Lemere CA, Xia W, Tseng HC, Zhou Y, Neve R, Ahlijanian MK, Tsai LH (2003) APP processing is regulated by cytoplasmic phosphorylation. J Cell Biol 163: 83-95.

Li M, Makkinje A, Damuni Z (1996) The myeloid leukemia-associated protein SET is a potent inhibitor of protein phosphatase 2A. J Biol Chem: 11059-11062.

Li X, Wu L, Corsa CA, Kunkel S, Dou Y (2009) Two mammalian MOF complexes regulate transcription activation by distinct mechanisms. Mol Cell 36: 290-301.

Liao YF, Wang BJ, Cheng HT, Kuo LH, Wolfe MS (2004) Tumor necrosis factor-alpha, interleukin-1beta, and interferon-gamma stimulate gamma-secretase-mediated cleavage of amyloid precursor protein through a JNK-dependent MAPK pathway. J Biol Chem 279: 49523-49532.

Lieber MR (2010) The mechanism of double-strand DNA break repair by the nonhomologous DNA end-joining pathway. Annu Rev Biochem 79: 181-211.

Lisby M, Rothstein R (2009) Choreography of recombination proteins during the DNA damage response. DNA Repair 8: 1068-1076.

Lopez-Lopez C, Dietrich MO, Metzger F, Loetscher H, Torres-Aleman I (2007) Disturbed cross talk between insulin-like growth factor I and AMP-activated protein kinase as a possible cause of vascular dysfunction in the amyloid precursor protein/presenilin 2 mouse model of Alzheimer's disease. J Neurosci 27: 824-831.

Luijsterburg MS, Dinant C, Lans H, Stap J, Wiernasz E, Lagerwerf S, Warmerdam DO, Lindh M, Brink MC, Dobrucki JW, Aten JA, Fousteri MI, Jansen G, Dantuma NP, Vermeulen W, Mullenders LH, Houtsmuller AB, Verschure PJ, van Driel R (2009) Heterochromatin protein 1 is recruited to various types of DNA damage. I Cell Biol 185: 577-586.

Mahaney BL, Meek K, Lees-Miller SP (2009) Repair of ionizing radiation-induced DNA double-strand breaks by non-homologous endjoining. Biochem J 417: 639-650.

Mimitou EP, Symington LS (2009) DNA end resection, many nucleases make light work. DNA Repair 8: 983-995.

Minopoli G, Stante M, Napolitano F, Telese F, Aloia L, De Felice M, Di Lauro R, Pacelli R, Brunetti A, Zambrano N, Russo T (2007) Essential roles for Fe65, Alzheimer amyloid precursor-binding protein, in the cellular response to DNA damage. J Biol Chem 282: $831-835$.

Moon SH, Nguyen TA, Darlington Y, Lu X, Donehower LA (2010) Dephosphorylation of gammaH2AX by WIP1, an important homeostatic regulatory event in DNA repair and cell cycle control. Cell Cycle 9: 2092-2096.

Müller T, Meyer HE, Egensperger R, Marcus K (2008) The amyloid precursor protein intracellular domain (AICD) as modulator of gene expression, apoptosis, and cytoskeletal dynamics-relevance for Alzheimer's disease. Prog Neurobiol 85: 393-406.

Murr R, Loizou JI, Yang YG, Cuenin C, Li H, Wang ZQ, Herceg Z (2006) Histone acetylation by Trrap-Tip60 modulates loading of repair proteins and repair of DNA double-strand breaks. Nat Cell Biol 8: $91-99$.
Murr R, Vaissière T, Sawan C, Shukla V, Herceg Z (2007) Orchestration of chromatin-based processes: mind the TRRAP. Oncogene 26: 5358-5372.

Muto S, Senda M, Akai Y, Sato L, Suzuki T, Nagai R, Senda T, Horikoshi M (2007) Relationship between the structure of SET/ TAF-Ibeta/INHAT and its histone chaperone activity. Proc Natl Acad Sci USA 104: 4285-4290.

Nakamura AJ, Rao VA, Pompier Y, Bonnier WM (2010) The complexity of phosphorylated H2AX foci formation and DNA repair assembly at DNA double-strand breaks. Cell Cycle 9: 389-397.

Nakaya T, Suzuki T (2006) Role of APP phosphorylation in FE65dependent gene transactivation mediated by AICD. Genes Cells 11: 633-645.

Narang H, Bhat N, Gupta S, Santra S, Choudhary RK, Kailash S, Krishna M (2009) Differential activation of mitogen-activated protein kinases following high and low LET radiation in murine macrophage cell line. Mol Cell Biochem 324: 85-91.

Nikolaev A, McLaughlin T, O'Leary DD, Tessier-Lavigne M (2009) APP binds DR6 to trigger axon pruning and neuron death via distinct caspases. Nature 457: 981-989.

Noon AT, Shibata A, Rief N, Löbrich M, Stewart GS, Jeggo PA, Goodarzi AA (2010) 53BP1-dependent robust localized KAP-1 phosphorylation is essential for heterochromatic DNA doublestrand break repair. Nat Cell Biol 12: 177-184.

Pandita TK, Richardson C (2009) Chromatin remodeling finds its place in the DNA double-strand break response. Nucleic Acids Res 37: 1363-1377.

Pardo B, Gómez-González B, Aguilera A (2009) DNA repair in mammalian cells, DNA double-strand break repair, how to fix a broken relationship. Cell Mol Life Sci 66: 1039-1056.

Perkinton MS, Standen CL, Lau KF, Kesavapany S, Byers HL, Ward M, McLoughlin DM, Miller CC (2004). The c-Abl tyrosine kinase phosphorylates the Fe65 adaptor protein to stimulate Fe65/amyloid precursor protein nuclear signaling. J Biol Chem 279: 22084-22091.

Reinhard C, Hébert SS, De Strooper B (2005) The amyloid-beta precursor protein, integrating structure with biological function. EMBO J 24: 3996-4006.

Robert F, Hardy S, Nagy Z, Baldeyron C, Murr R, Déry U, Masson JY, Papadopoulo D, Herceg Z, Tora L (2006) The transcriptional histone acetyltransferase cofactor TRRAP associates with the MRN repair complex and plays a role in DNA double-strand break repair. Mol Cell Biol 26: 402-412.

Russo T, Faraonio R, Minopoli G, De Candia P, De Renzis S, Zambrano N (1998) Fe65 and the protein network centered around the cytosolic domain of the Alzheimer's beta-amyloid precursor protein. FEBS Lett 434: 1-7.

Sapountzi V, Logan IR, Robson CN (2006) Cellular functions of TIP60. Int J Biochem Cell Biol 38: 1496-1509.

Seo SB, McNamara P, Heo S, Turner A, Lane WS, Chakravarti D (2001) Regulation of histone acetylation and transcription by INHAT, a human cellular complex containing the set oncoprotein. Cell 104: $119-130$.

Seo SB, Macfarlan T, McNamara P, Hong R, Mukai Y, Heo S, Chakravarti D (2002) Regulation of histone acetylation and transcription by nuclear protein pp32, a subunit of the INHAT complex. J Biol Chem 277: 14005-14010.

Sharma GG, So S, Gupta A, Kumar R, Cayrou C, Avvakumov N, Bhadra U, Pandita RK, Porteus MH, Chen DJ, Cote J, Pandita TK (2010) MOF and histone H4 acetylation at lysine 16 are critical for DNA damage response and double-strand break repair. Mol Cell Biol 30: 3582-3595.

Słomnicki LP, Leśniak W (2008) A putative role of the amyloid precursor protein intracellular domain (AICD) in transcription. Acta Neurobiol Exp (Wars) 68: 219-228.

Sondag CM, Combs CK (2004) Amyloid precursor protein mediates proinflammatory activation of monocytic lineage cells. $J$ Biol Chem 279: $14456-14463$.

Squatrito M, Gorrini C, Amati B (2006) Tip60 in DNA damage response and growth control: many tricks in one HAT. Trends Cell Biol 16: 433-442.

Stante M, Minopoli G, Passaro F, Raia M, Vecchio LD, Russo T (2009) Fe65 is required for Tip60-directed histone $\mathrm{H} 4$ acetylation at DNA strand breaks. Proc Natl Acad Sci USA 106: 5093-5098.

Sun Y, Xu Y, Roy K, Price BD (2007) DNA damage-induced acetylation of lysine 3016 of ATM activates ATM kinase activity. Mol Cell Biol 27: 8502-8509.

Sun Y, Jiang X, Xu Y, Ayrapetov MK, Moreau LA, Whetstine JR, Price BD (2009) Histone H3 methylation links DNA damage detection to activation of the tumour suppressor Tip60. Nat Cell Biol 11: 1376-1382.

Sun Y, Jiang X, Price BD (2010) Tip60, connecting chromatin to DNA damage signaling. Cell Cycle 9: 930-936.

Taipale M, Rea S, Richter K, Vilar A, Lichter P, Imhof A, Akhtar A (2005) hMOF histone acetyltransferase is required for histone $\mathrm{H} 4$ lysine 16 acetylation in mammalian cells. Mol Cell Biol 25: 67986810 . 
Tamayev R, Zhou D, D'Adamio L (2009) The interactome of the amyloid beta precursor protein family members is shaped by phosphorylation of their intracellular domains. Mol Neurodegener 4: 28.

Thomas T, Loveland KL, Voss AK (2007) The genes coding for the MYST family histone acetyltransferases, Tip60 and Mof, are expressed at high levels during sperm development. Gene Expr Patterns 7: $657-665$.

Thomas T, Dixon MP, Kueh AJ, Voss AK (2008) Mof (MYST1 or KAT8) is essential for progression of embryonic development past the blastocyst stage and required for normal chromatin architecture. Mol Cell Biol 28: 5093-5105.

Telese F, Bruni P, Donizetti A, Gianni D, D’Ambrosio C, Scaloni A, Zambrano N, Rosenfeld MG, Russo T (2005) Transcription regulation by the adaptor protein Fe65 and the nucleosome assembly factor SET. EMBO Rep 6: 77-82.

Varela I, Pereira S, Ugalde AP, Navarro CL, Suárez MF, Cau P, Cadiñanos J, Osorio FG, Foray N, Cobo J, de Carlos F, Lévy N, Freije JM, López-Otín C (2008) Combined treatment with statins and aminobisphosphonates extends longevity in a mouse model of human premature aging. Nat Med 14: 767-772.
Vázquez MC, Vargas LM, Inestrosa NC, Alvarez AR (2009) c-Abl modulates AICD dependent cellular responses, transcriptional induction and apoptosis. J Cell Physiol 220: 136-143.

von Rotz RC, Kohli BM, Bosset J, Meier M, Suzuki T, Nitsch RM, Konietzko U (2004) The APP intracellular domain forms nuclear multiprotein complexes and regulates the transcription of its own precursor. J Cell Sci 117: 4435-4448.

Williamson R, Scales T, Clark BR, Gibb G, Reynolds CH, Kellie S, Bird IN, Varndell IM, Sheppard PW, Everall I, Anderton BH (2002) Rapid tyrosine phosphorylation of neuronal proteins including tau and focal adhesion kinase in response to amyloid-beta peptide exposure, involvement of Src family protein kinases. I Neurosci 22: $10-20$.

Zambrano N, Minopoli G, de Candia P, Russo T (1998) The Fe65 adaptor protein interacts through its PID1 domain with the transcription factor CP2/LSF/LBP1. J Biol Chem 273: 20128-20133.

Zarębski M, Wiernasz E, Dobrucki JW (2009) Recruitment of heterochromatin protein 1 to DNA repair sites. Cytometry A 75: 619-625. 